\title{
Inertia force effect on longitudinal vibrations of underground pipelines
}

\author{
Barna Rahkmankulova ${ }^{1}$, Sayibdjan Mirzaev ${ }^{1}$, Sharafidin Aynakulov ${ }^{1}$, Rakhmatjon \\ Khusainov $^{2}$, and Saparboy Khusainov ${ }^{3}$ \\ ${ }^{1}$ Tashkent Institute of Irrigation and Agricultural Mechanization Engineers, Tashkent, Uzbekistan \\ ${ }^{2}$ Tashkent Institute of Mechanics and Seismic Stability of Structures named after M.T.Urazbaev, \\ Tashkent, Uzbekistan \\ ${ }^{3}$ Bauman Moscow State Technical University, Moscow, Russia
}

\begin{abstract}
The effect of the inertia term on the longitudinal displacements of an underground pipeline is shown for various cases of pipe fastening when a seismic wave propagates along its axis. The problem is solved by analytical and numerical methods. The pipe-soil interaction is assumed to be elastic (shear stress generated in soil is proportional to the relative displacement between the pipe and soil).
\end{abstract}

\section{Introduction}

Damage to underground pipelines during an earthquake is associated with a combination of different types of hazards: permanent soil deformations (landslides, liquefaction, and seismic settlement) and wave propagation effect. The latter is characterized by transient deformation and curvature in the ground due to the travelling wave effect. For the analysis of wave propagation, Rashidov and Newmark $[1,2]$ proposed a simple procedure taking into account one travelling wave with an undamped (travelling) waveform. Therefore, the maximum axial deformation of the pipe is the same as the maximum axial deformation of the ground.

However, the above procedures consider infinite pipe lengths and therefore do not consider their effective length and construction works (constraint conditions). In [3], analytical relations were developed for a pipe of finite length subjected to various combinations of boundary conditions (i.e., the free end, fixed or elastic boundary) for pipelines of different lengths. In 1962, T. Rashidov proposed a differential equation for an underground pipeline, which became the basis of the dynamic theory. Rashidov [1, 3], De Martino et al. [4], and Corrado et al. [5] developed models for the pipe-soil interaction, taking into account the limited length of the pipeline. Assuming a linear elastic model of ground motion and ignoring the slip at the pipe-soil contact interface, the models analyze the dynamic behaviour of a finite-length pipeline taking into account the boundary conditions at the ends. The pipeline was supposed to be continuous; that is, any fluctuations between the characteristics (parameters) of the pipeline and its joints are considered insignificant. A.A. Ilyushin and T. Rashidov [6] proposed a visco-elastoplastic model of the interaction of an underground structure with soil. 
In the works [7-8], the use of different mechanical mathematical models was analyzed, and several urgent problems of underground and ground structures were solved.

The study in [9] shows the comparison between the methods of Crank-Nicholson, McCormack, and Courant-Friedrichs-Lewy (explicit scheme). It shows the accuracy of the explicit scheme relative to other methods when solving discontinuous problems of underground pipeline vibrations. In that study, the problem is solved by the finite difference method in an explicit scheme. Careful numerical calculations are performed to prevent unwanted vibrations near the discontinuity (the deformation wave front) [10-12].

In [10-14], the effect of the coefficients of elasticity, viscosity, and plasticity of the pipeline contact interaction with soil on the stress-strain state of an underground pipeline is studied in detail. In $[5,12]$, the longitudinal vibration of an underground pipeline with and without inertia forces is compared for an infinite pipeline and a pipeline of finite length with rigid fixing at the ends.

Seismic analysis of the pipeline interaction with surrounding soil was performed. The pipe-soil interaction is assumed to be elastic (shear stress generated in soil is proportional to the relative displacement between the pipe and soil). The effect of the inertial term on the longitudinal displacements of an underground pipeline in various cases of pipe fastening when a seismic wave propagates along its axis is shown in the article. The problem is solved by analytical and numerical methods [15-17].

\subsection{Statement of problem}

Consider a pipe of length $L$ for which the equation of motion is written in the following form:

$$
m_{p} \frac{\partial^{2} u}{\partial t^{2}}-E F \frac{\partial^{2} u}{\partial x^{2}}+K\left(u-u_{g}\right)=0
$$

where $x$ and $t$ are the spatial and temporal variables; $u$ and ${ }^{u} g$ are the axial displacements of the pipe and soil, respectively; $m_{p}$ is the mass per unit length; $E$ is the Young's modulus of the pipe material; $F=\pi s(D-s)$ is the cross-sectional area of the pipe, where $S$ and $D$ are the thickness and outer diameter of the pipe, respectively; $K=k_{X} \pi D$, where ${ }^{k} X$ is the coefficient of elastic interaction in the "pipe - soil" system. Ground motion is given by a sine wave. Soil displacement parallel to the pipe can be written as:

$$
u_{g}=\left\{\begin{array}{c}
A \cdot \sin \omega\left(t-\frac{x}{C_{p}}\right), \text { if } t>\frac{x}{C_{p}}, \\
0, \quad \text { if not }
\end{array}\right.
$$

where $A$ is the maximum displacement of soil, $\omega$ is the vibration frequency of the seismic wave, determined by formula $\omega=2 \pi v$, where $v$ is the frequency; $C_{p}$ is the velocity of seismic wave propagation.

Suppose that at the beginning of motion, the underground pipeline was at rest and in an undeformed state, then, the initial conditions are formulated as follows: 


$$
\left.u\right|_{t=0}=0,\left.\dot{u}\right|_{t=0}=0
$$

Let us take the boundary conditions in a more general form, that is, let us assume that both ends of the pipeline are flexibly fixed, and then the boundary condition has the form:

$$
\begin{aligned}
& \left.E \cdot F \cdot \frac{\partial u}{\partial x}\right|_{x=0}=\left.k_{0} \cdot u\right|_{x=0} \\
& \left.E \cdot F \cdot \frac{\partial u}{\partial x}\right|_{x=L}=-\left.k_{1} \cdot u\right|_{x=L}
\end{aligned}
$$

Here $k_{0}$ and $k_{1}$ are the yielding coefficients at the left and right boundaries, respectively.

This boundary condition is universal, by varying values of $k_{0}$ and $k_{1}$ we obtain different boundary conditions. If the values of $k_{0}$ and $k_{1}$ are zero, then we obtain the free ends; if these values are equal to infinity, then we get the rigid ends.

With the movement of an underground pipeline, the absolute movement of the pipe is equal to the sum of the so-called relative and transfer movement of the pipe. For the relative displacement, we take the pipe displacement relative to the soil, and the transfer displacement of soil is determined by

$$
u=u_{r}+u_{e}, \quad u_{e}=u_{g}
$$

Consider equation (1) without inertial term generated by the relative displacement of the pipe. Equation (1) takes the following form:

$$
m_{p} \frac{\partial^{2} u_{g}}{\partial t^{2}}-E F \frac{\partial^{2} u}{\partial x^{2}}+K\left(u-u_{g}\right)=0
$$

Now let us consider the same problem, but without the absolute inertial term of the pipe. Then, equation (1) takes the following form [5]:

$$
\frac{\partial^{2} u}{\partial x^{2}}-\frac{K}{E F}\left(u-u_{g}\right)=0 .
$$

\section{Materials and Methods}

Equation (1) is solved, taking into account the initial conditions (3) and boundary conditions (4), using an explicit finite-difference scheme, with the choice of the ratio of increment steps in coordinate and time as $\Delta x / \Delta t=a$, where $a=\sqrt{E \cdot F / m_{p}}$ is the velocity of wave propagation in the pipeline. The calculations were performed at $\Delta x=0.01$ m.

The general solution of equations (5) and (6) has the form: 


$$
u=C_{1} u_{1}+C_{2} u_{2}+\bar{u}
$$

where $C_{1}$ and $C_{2}$ are the constants; $u_{1}$ and $u_{2}$ are two independent solutions of the homogeneous equation; $\bar{u}$ is the particular solution of equation (5) and (6).

Let the ground moves according to formula (2). Then, solving the corresponding homogeneous equation, we obtain the general solution of equations (5) and (6) in the form

$$
u=C_{1} e^{X \sqrt{K / E F}}+C_{2} e^{-X \sqrt{K / E F}}+B A \sin \omega\left(t-\frac{x}{C_{P}}\right)
$$

where $B$ is an arbitrary constant, determined for equation (5) by the following formula:

$$
B=\frac{\left(m_{p} \cdot \omega^{2}+K\right) / E F}{K / E F+\left(\omega / C_{P}\right)^{2}}
$$

and for equation (6) it is determined by [5]:

$$
B=\frac{K / E F}{K / E F+\left(\omega / C_{P}\right)^{2}}
$$

When considering a pipe of finite length $L$, the constants $C_{1}$ and $C_{2}$, entering (8), are nonzero; their corresponding values are calculated depending on the boundary conditions (4).

The above systems are quasi-static: it is easy to determine the values of the two constants for the specified boundary conditions changing the values of $t$.

The axial displacement and the pipe deformation for the analyzed boundary conditions can be easily calculated.

Consider a solid steel pipeline with the following pipe and soil characteristics.

Steel pipe characteristics are diameter $D=0.5 \mathrm{~m}$; thickness $\mathrm{s}=0.01 \mathrm{~m}$; Young's modulus $\mathrm{E}=2.010^{8} \mathrm{kN} / \mathrm{m}^{2}$; length $\mathrm{L}=100.0 \mathrm{~m}$; density $\rho=7800.0 \mathrm{~kg} / \mathrm{m}^{3}$.

Soil characteristics are elastic soil resistance $k_{x}=(0.5-4) 10^{7} \mathrm{~N} / \mathrm{m}^{3}$; wave propagation velocity $C_{p}=500.0 \mathrm{~m} / \mathrm{s}$; period of basic vibrations $T=0.3 \mathrm{~s}$; vibration amplitude $A=0.004$ $\mathrm{m}$.

\section{Results and Discussion}

First, we analyze the solution to the differential equation (1). Underground pipeline with boundary conditions, its left end is rigidly fixed, and the right end is free. That is, with the boundary conditions (4) $\mathrm{k}_{0}=\infty$ and $\mathrm{k}_{1}=0$, equation (1) is solved using the numerical finite difference method [18]. The results obtained are shown in Figures 1 and 2. Figure 1 shows the absolute motion of an underground pipeline 100 meters long in time for 10, 40, 90meter sections of the pipeline. From this figure, it can be seen that with the distance from the fixed end, the displacement up to the distance of 30 meters increases faster, and after this point, it becomes slower. Figure 2 shows the change in displacement along the pipeline 
for different points in time, that is, at $0.1,0.2,0.3 \mathrm{~s}$. At $0.1 \mathrm{~s}$, a travelling wave of the velocity of $500 \mathrm{~m} / \mathrm{s}$ will not reach the right boundary of an underground pipeline 100 meters long. Therefore, as seen from Fig. 2, the underground pipeline is moving up to the point of 70 meters. The wave has not yet reached the 70-100 meter section; therefore, this section of the pipeline is at rest. Before the travelling wave reaches the right boundary, the maximum displacement increases, and, reaching the maximum, it remains approximately unchanged. From figure 2, it can be seen that the pipe is stretched when the wave reaches the second end of the pipeline; after reaching the second end, the situation changes: one part is stretched, and the other part is compressed; such a point is determined as an imaginary rest.

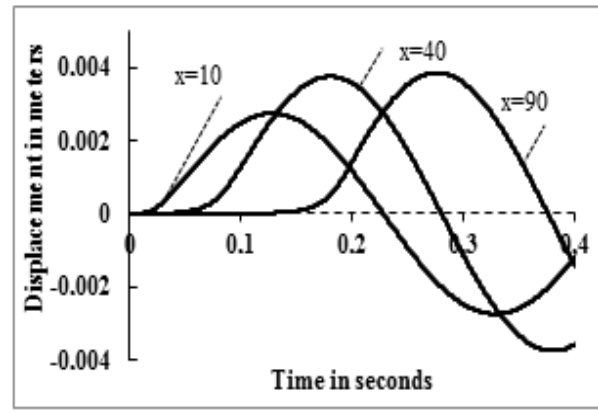

Fig. 1. Change in displacement with time when boundary condition are: the left end is rigidly fixed, and the right end is free.

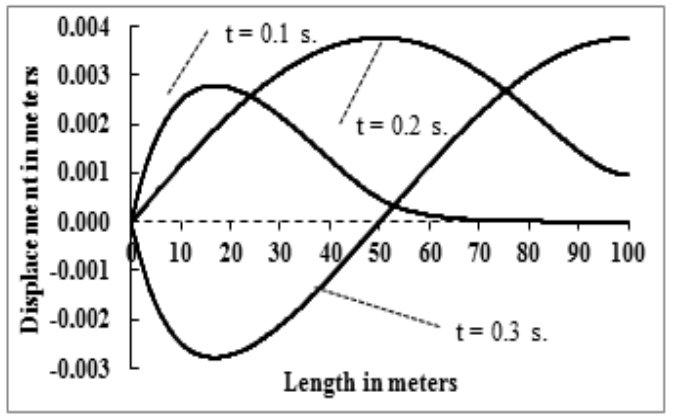

Fig. 2. Change in displacement along the pipeline length when boundary condition are: the left end is rigidly fixed, and the right end is free.

Figure 3 shows the change in deformation in the time range $0-0.4$ seconds, that is, one period of ground vibration. The deformation reaches its maximum value at the rigidly fixed end. The maximum deformation value decreases rapidly by approximately 3.2 times over the interval from 0 to 10 meters. In the 10 - 90 meter section, the deformation decreases by approximately1.75 times slower. From the results given above, it can be concluded that the dangerous zones under travelling harmonical ground motion are the zones located closer to the rigidly fixed end of the underground pipeline. Investigation of the consequences of the Tashkent earthquake, 1966, revealed damage to underground pipelines in the vicinity of wells, pumping stations and abrupt turns [19]. This proves the reliability of our results. Figure 4 shows the change in deformation along the underground pipeline at the points of time $0.1,0.2$ and 0.3 seconds; this also confirms the above conclusions.

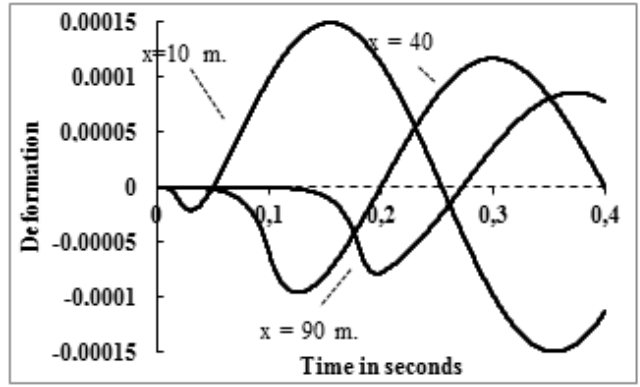

Fig. 3. Change in deformation with time at different sections of the pipeline. The left end is rigidly fixed, and the right end is free.

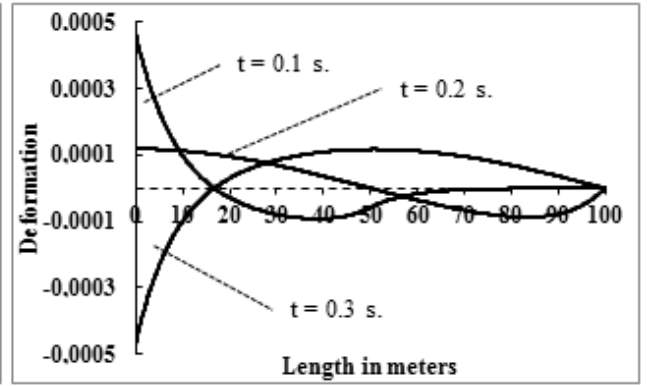

Fig. 4. Change in deformation along the pipeline at different time points when the left end is rigidly fixed, and the right end is free. 

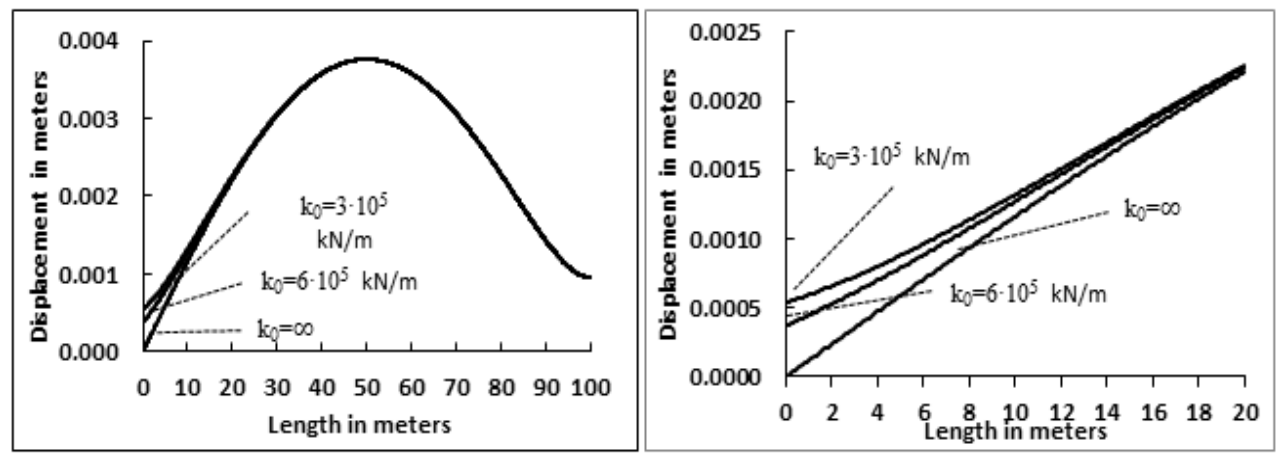

Fig. 5 and 6. Change in displacement along the pipeline at different values of the yielding coefficient of the left boundary at the moment $t=0.2 \mathrm{~s}$. (The right end is free)

Figure 7 shows a graph of the change in deformation of an underground pipeline with time under the following boundary conditions: the left end is flexibly fixed, and the right end is free. Figure 7 shows that with an increase in the yielding coefficient, the maximum deformation value increases. Figure 8 shows the change in deformation along the underground pipeline length at $0.2 \mathrm{~s}$ at different values of the yielding coefficient of fastenings. The deformation differs for different values of the yielding coefficient of fastenings near the boundary, but in other sections, the values are the same.

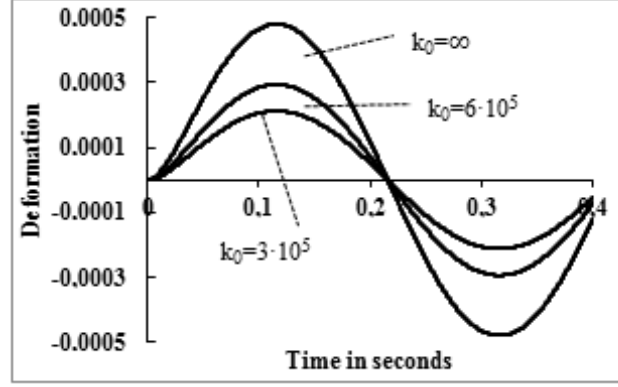

Fig. 7. Change in deformation with time at the left end under different values of the yielding coefficient of fastenings on the left boundary

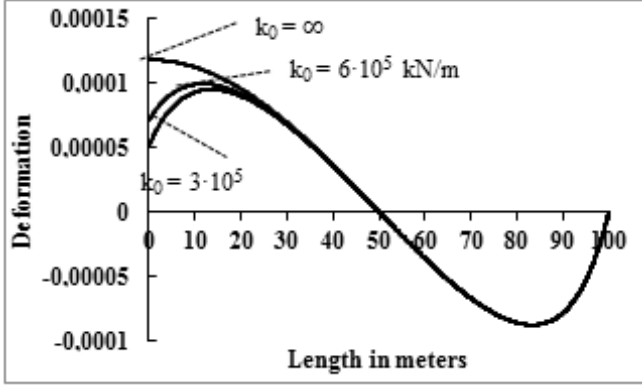

Fig. 8. Change in deformation along the pipeline under different values of the yielding coefficient of the left boundary at time $\mathrm{t}=0.2 \mathrm{~s}$.

Figure 9 shows the change in deformation with time. A comparison of the solution of equations (1), (5), and (6) gives the following conclusions; from the graphs, it is seen that the differences are observed only at the beginning of the motion, and then they coincide. Figure 10 shows the change in deformation along the length; it is seen that only in the case when the travelling wave reaches the right end of the pipeline at the wave front there is a difference between the solutions to the equation with and without inertia. With Figs. 9 and 10 , it can be seen that the results coincide without considering the absolute and relative accelerations. Therefore, in the following discussions, we only compare the two cases with and without absolute acceleration. 


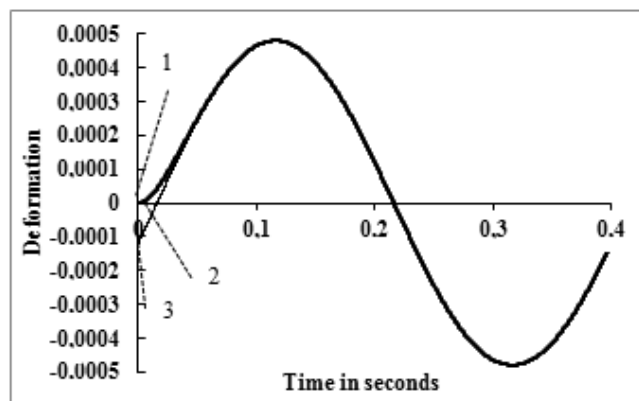

Fig. 9. Change in deformation with time: 1 is with absolute acceleration; 2 is without relative acceleration; 3 is without absolute acceleration.

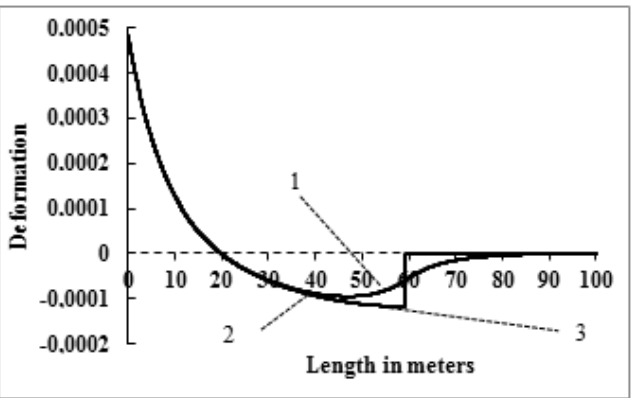

Fig. 10. Deformation change along the length $(\mathrm{t}=0.185 \mathrm{~s}$.$) : 1$ is with absolute acceleration; 2 is without relative acceleration; 3 is without absolute acceleration.

For the problems in seismic dynamics, the maximum values of displacement and deformation are the most interesting factors. Therefore, below we compare the problems with and without inertia in the maximum deformations. We will show whether the results with and without inertia always coincide.

Figure 11 shows the change in maximum deformation with a change in the yielding coefficient at the left end when the right end is free. With a yield coefficient in the range of $0-10,10^{4} \mathrm{kN} / \mathrm{m}$, the maximum deformation does not change; when the coefficient is greater than $10^{5} \mathrm{kN} / \mathrm{m}$, the maximum deformation increases monotonically. Comparison of results is shown in Table 1.

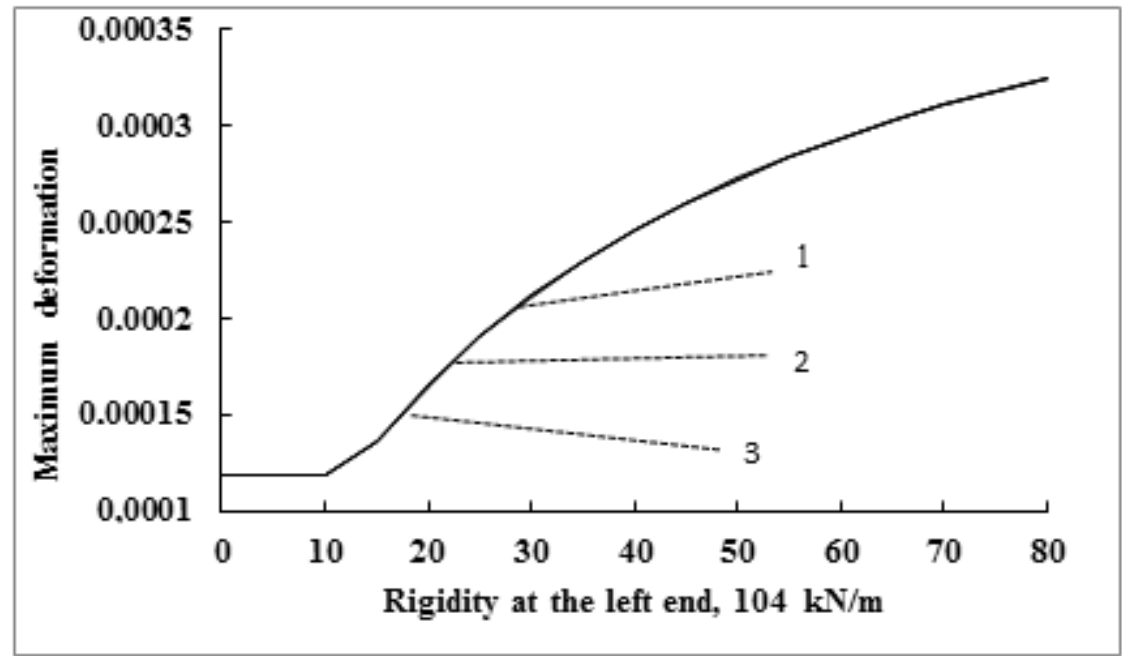

Fig. 11. Change in the maximum deformation with a change in the yielding coefficient at the left end: 1 is with absolute acceleration; 2 is without relative acceleration; 3 is without absolute acceleration. 
Table 1. Comparison of the results of equations (1), (5), and (6) at different values of the yielding coefficient at the left end of the pipeline

\begin{tabular}{|c|c|c|c|}
\hline & \multicolumn{3}{|c|}{ Maximum deformation value } \\
\hline $\begin{array}{c}\text { Rigidity at } \\
\text { the left end, } \\
\mathbf{1 0} \mathbf{~} \mathrm{kN} / \mathrm{m}\end{array}$ & $\begin{array}{c}\text { With absolute } \\
\text { acceleration, } 10^{-4}\end{array}$ & $\begin{array}{c}\text { Without relative } \\
\text { acceleration, } 10^{-4}\end{array}$ & $\begin{array}{c}\text { Without absolute } \\
\text { acceleration, } 10^{-4}\end{array}$ \\
\hline 10 & 1.1810998233295 & 1.17970521012018 & 1.17896400276093 \\
\hline 40 & 2.45874554583607 & 2.45845615643537 & 2.45691151140013 \\
\hline
\end{tabular}

Figure 12 shows the change in maximum deformation depending on the coefficient of elastic interaction. As seen from this figure, the results obtained with and without this coefficient coincide; only in the range from $0.5 \cdot 10^{4} \mathrm{kN} / \mathrm{m}$ to $10^{4} \mathrm{kN} / \mathrm{m}$ there is a slight difference. Table 2 shows the maximum deformation values for some values of the elastic interaction coefficient. Based on the data from Table 2, we can compare the difference between the results obtained with absolute acceleration, without absolute acceleration and without relative acceleration.

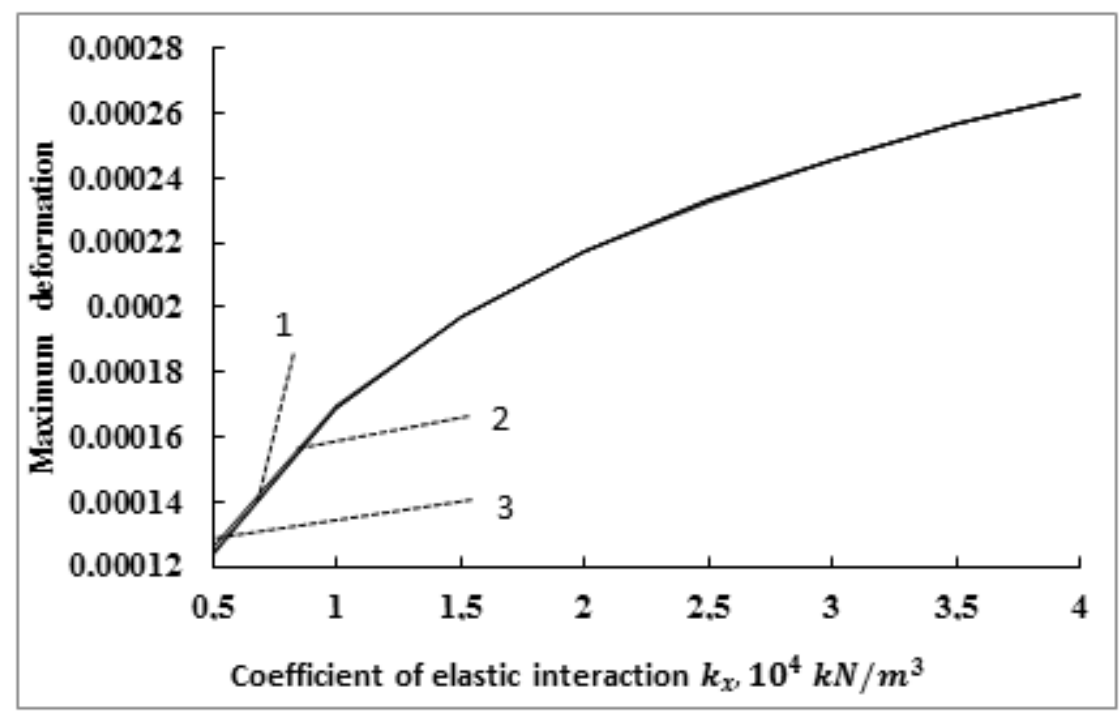

Fig. 12. Change in maximum deformation depending on the coefficient of elastic interaction of the "pipe-soil" system: 1 is with absolute acceleration; 2 is without relative acceleration; 3 - is without absolute acceleration.

Maximum deformation changes with a change in the yielding coefficient at the left end.

Table 2. Comparison of the results of equations (1), (5), and (6) at different values of the coefficient of elastic interaction of the "pipe-soil" system

\begin{tabular}{|c|c|c|c|}
\hline \multirow{2}{*}{$\mathrm{k}_{\mathrm{x}}, 10^{7} \mathrm{~N} / \mathrm{m}^{3}$} & \multicolumn{3}{|c|}{ Maximum deformation value } \\
\cline { 2 - 4 } & $\begin{array}{c}\text { With absolute } \\
\text { acceleration, } 10^{-4}\end{array}$ & $\begin{array}{c}\text { Without relative } \\
\text { acceleration, } 10^{-4}\end{array}$ & $\begin{array}{c}\text { Without absolute } \\
\text { acceleration, } 10^{-4} .\end{array}$ \\
\hline 0.5 & 1.25944489837928 & 1.24436308824335 & 1.23968678785595 \\
\hline 3 & 2.45874554583607 & 2.45845615643537 & 2.45691151140013 \\
\hline
\end{tabular}

Figure 13 shows a comparative graph of the maximum deformation value depending on the period of ground vibration under seismic impact at small values of the period, from 0.1 $\mathrm{s}$ to $0.3 \mathrm{~s}$. The difference in results of the above-mentioned problems is shown in Table 3. 
In the case of account for inertia in this interval, the difference is more pronounced than without inertia.

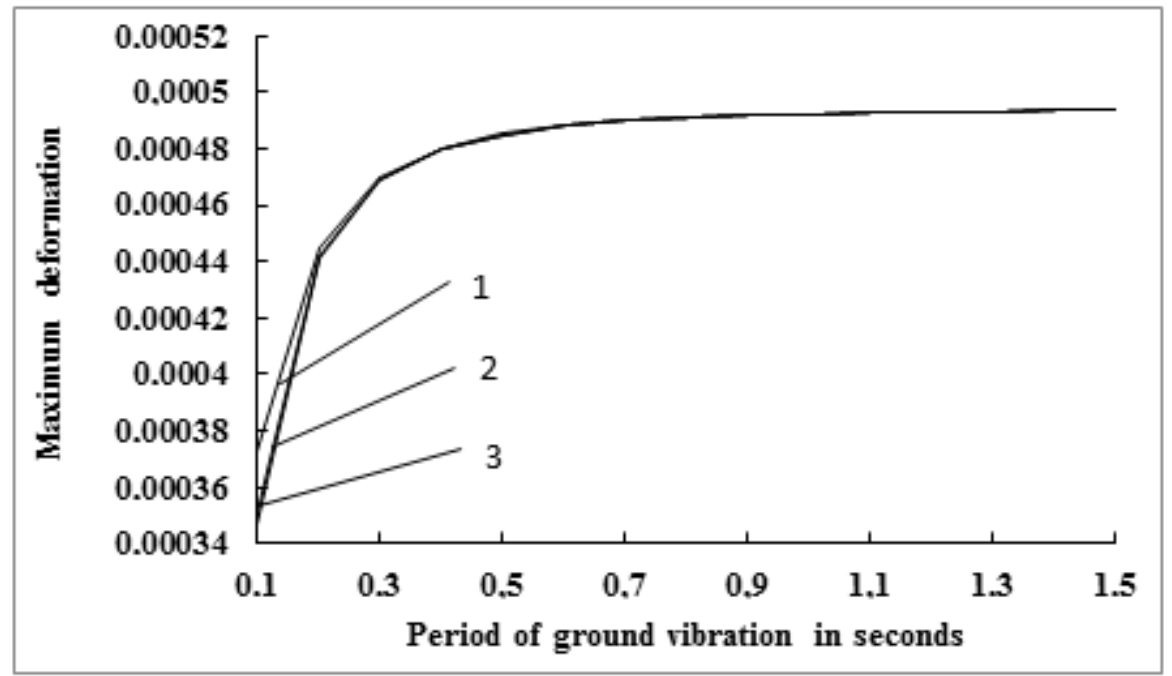

Fig. 13. Change in maximum deformation depending on the period of ground vibration: 1 is with absolute acceleration; 2 is without relative acceleration; 3 is without absolute acceleration.

Table 3. Comparison of the results of equations (1), (5), and (6) at different values of the period of ground vibration

\begin{tabular}{|c|c|c|c|}
\hline & \multicolumn{3}{|c|}{ Maximum deformation value } \\
\hline $\begin{array}{c}\text { Ground vibration } \\
\text { period in } \\
\text { seconds }\end{array}$ & $\begin{array}{c}\text { With absolute } \\
\text { acceleration, } 10^{-4}\end{array}$ & $\begin{array}{c}\text { Without relative } \\
\text { acceleration, } 10^{-4}\end{array}$ & $\begin{array}{c}\text { Without absolute } \\
\text { acceleration, } 10^{-4} .\end{array}$ \\
\hline 0.2 & 4.45224164558535 & 4.4233544991188 & 4.41225866045733 \\
\hline 0.5 & 4.85039138183247 & 4.85156531550118 & 4.84961405753406 \\
\hline
\end{tabular}

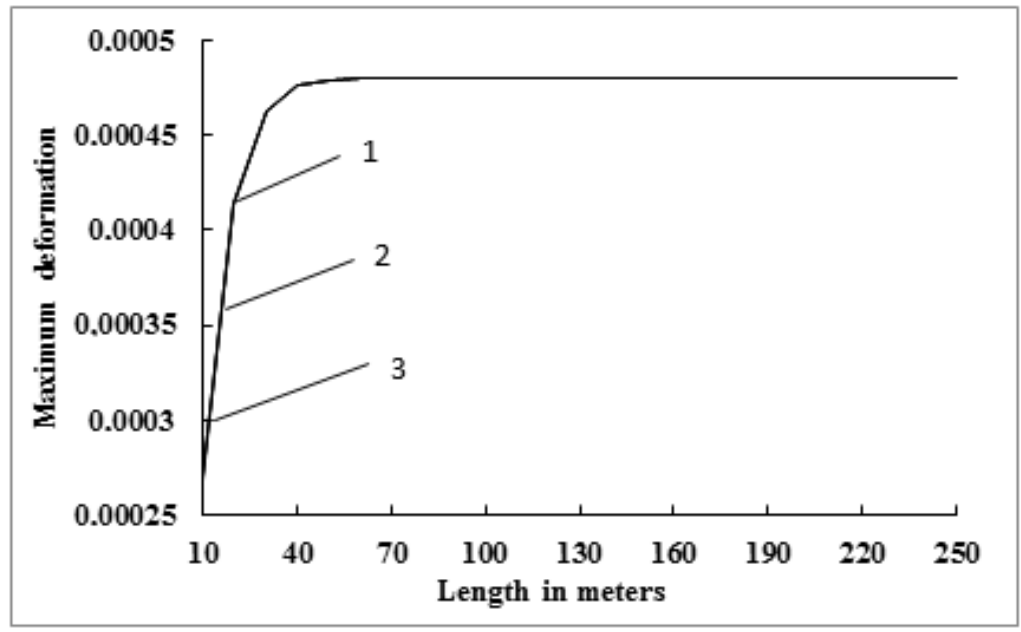

Fig. 14. Change in maximum deformation depending on the length of the pipeline: 1 is with absolute acceleration; 2 is without relative acceleration; 3 is without absolute acceleration. 
Fig. 14 shows a comparative graph of the maximum deformation value depending on the length of the pipeline. Here the difference in the graphs is not seen. This result is obtained for a section of the underground pipeline 100 meters long and the vibration period of 0.4 seconds. The travelling harmonic wave will reach its maximum value in a quarter of the vibration period. At a wave propagation velocity taken as $500 \mathrm{~m} / \mathrm{s}$, the wave will reach 50 meters in 0.1 seconds. This can explain that the maximum deformation value does not change for the underground pipelines longer than $T \cdot C_{p} / 4$ (in our case, it is 50 meters).

From the comparison of the above results, several conclusions can be drawn.

\section{Conclusions}

Before the travelling harmonic wave reaches the second boundary of the pipeline, the difference between the result with and without the inertial term of equation (1) is seen only in the wave front.

The change in the yielding coefficient of the fastenings at the boundaries does not contribute to the agreement of results with and without inertia.

For any values of the coefficient of elastic interaction, the results with and without inertia coincide with each other by a miniscule difference.

The change in the vibration period is observed only from $0.1 \mathrm{~s}$ to $0.3 \mathrm{~s}$. In periods greater than $0.3 \mathrm{~s}$ there is a difference in the maximum deformation values. The values coincide.

We can conclude that it is enough to consider the quasi-static problem for a problem of elastic interactions to determine the maximum value.

When calculating the longitudinal vibrations of an underground pipeline, with both ends flexibly fixed, the inertia force of the pipe can be ignored; this makes it possible to solve the problem in a simplified form.

\section{References}

1. T. Rashidov, Differential equation of underground pipeline vibrations during an earthquake, Tashkent, Reports of the Academy of Sciences of Uzbekistan, 9, pp. 1012, (1962).

2. Newmark N. M., Problems in wave propagation in soil and rocks, In Proceedings of the International Symposium on Wave Propagation and Dynamic Properties of Earth Materials, University of New Mexico Press, pp. 7-26, (1967)

3. Rashidov T. Calculation of underground pipelines of finite length under short-term seismic load, Tashkent, Reports of the Academy of Sciences UzR, 4, pp. 13-16, (1963).

4. Martino G.D, D'Acunto B., Fontana N., and Giugni M. Dynamic response of continuous buried pipes in seismic areas, In ASCE Pipelines Conference, (2006)

5. Virginia C., Acunto B., Fontana N., and Giugni M. Inertial Effects on Finite Length Pipe Seismic Response, MPE, p.14, (2012)

6. T.Rashidov, Dynamic theory of seismic stability of complex systems of underground structures, Tashkent: Fan, p.180, (1973)

7. M.M.Mirsaidov, T.Z.Sultanov, Assessment of stress-strain state of earth dams with allowance for non-linear strain of material and large strains, Mag. Civ. Eng., 49, pp. 73-82, (2014). 
8. K.S.Sultanov, N.I.Vatin, Wave Theory of Seismic Resistance of Underground Pipelines, Appl. Sci., 11 (4), https://doi.org/10.3390/app11041797, (2021).

9. V. Corrado, B. D'Acunto, N. Fontana, and M. Giugni, Estimation of dynamic strains in finite end-constrained pipes in seismic areas, Mathematical and Computer Modelling, 49(3-4), pp. 789-797, (2009).

10. R.B.Khusainov. On the issue of considering the wave entrainment of energy in the problems of calculating underground main pipelines, J. Problems of Mechanics, 4, pp. 107-111, (2018).

11. R.B.Khusainov. Longitudinal deformation wave in buried pipeline subject to viscoelastic interaction with soil, Soil Mechanics and Foundation Engineering, Springer 56, pp. 420-426, (2020)

12. R.B.Khusainov, Assessment of the inertia force effect on the longitudinal vibrations of underground pipelines, J. Problems of Mechanics, 4, pp. 72-75, (2017)

13. R.B.Khusainov, Seismodynamics of underground pipelines during visco-elasticplastic interaction with soil, IJARSET, 7(1) pp. 12468-12474, (2020)

14. R.B.Khusainov, analysis of longitudinal vibrations of an underground pipeline under elastic and viscoelastic interactions with soil under various boundary conditions, J. Problems of Mechanics, 1. pp. 86-90, (2017)

15. M.Yusupov, B.O.Rahkmankulova and Sh.A.Aynakulov. Numerical solutions of the problem of salt-transfer in soils, E3S Web of Conferences (Tashkent) (2019)

16. B.O.Rakhmankulova, K.Djamalov, N.X.Noraliyev, and Sh.K.Ziyaeva, Mathematical model of salt balance Stress-deformed state of ore-totropic spherical shell weakened by two circular holes. International Conference on Materials Physics, Building Structures and Technologies in Construction, Industrial and Production Engineering, Russia, (2020).

17. S.Mirzaev, I.Kholmatova, G.Shodmanova, M.Yusupov, and K.Kubyashev. Numerical modeling of two-dimensional two-phase filtration under frontal drive, International Conference "Construction Mechanics, Hydraulics and Water Resources Engineering" Tashkent, (2020)

18. A.A.Samarskiy, Theory of difference schemes. Moscow: Nauka, Physical-Math. Lit., p.616, (1989)

19. Tashkent earthquake on April 26, 1966, Tashkent: FAN Publishing house, p. 672, (1971) 